\title{
Carbon source utilisation by Bordetella bronchiseptica
}

\author{
BALBINA J. PLOTKIN and D.A. BEMIS* \\ Department of Microbiology, College of Osteopathic Medicine, Midwestern University, Downers Grove, $1 \mathrm{~L}$ and \\ * Department of Comparative Medicine, College of Veterinary Medicine, University of Tennessee, Knoxville, \\ TN, USA
}

\begin{abstract}
Bordetella bronchiseptica isolates utilised tricarboxylic acid cycle intermediates succinate, citrate, $\alpha$-ketoglutarate, fumarate, lactate and oxalo-acetate; the organic acids pyruvate, acetate and lactate; and the amino acids proline, glutamate, glutamine and tyrosine - as sole sources of carbon and energy. The inability of $B$. bronchiseptica isolates, representing the three phase types and from different animal hosts, to utilise carbohydrates and sugar alcohols as sole carbon and energy sources was confirmed and extended. The influence of the carbon substrate on doubling time, piliation, flagellation, motility, capsule production and adherence to mammalian cells was also measured.
\end{abstract}

\section{Introduction}

Bordetella bronchiseptica is a gram-negative, obligately aerobic bacillus that colonises the ciliated respiratory epithelium of a broad range of animals. It is the aetiological agent of kennel cough in dogs and atrophic rhinitis in swine, and has been implicated as a cause of respiratory tract disease in several other animal species, including man [1-4]. Although the survivability of $B$. bronchiseptica in low-nutrient conditions has been investigated $[5,6]$, there is little information on the carbon and energy requirements of the organism, or on whether carbon source requirements and ultrastructural characteristics vary between strains from different host species.

It has been proposed that $B$. bronchiseptica is asaccharolytic [7-12]. However, this classification is based on the singular finding that it is unable, aerobically, to use glucose as a sole carbon and energy source. In addition to the lack of information on the utilisation of sugars and sugar alcohols for growth, the literature conflicts with regard to the ability of $B$. bronchiseptica to utilise amino acids or organic acids as sole sources of carbon and energy for growth [9-12]. Ulrich and Needham [12] reported that the organism required a mixture of amino acids for growth, whereas Proom [9] found that some isolates were capable of using pyruvate or glutamate as sole carbon and energy sources.

Received 27 Aug. 1997; revised version accepted 12 Jan. 1998.

Corresponding author: Dr B.J. Plotkin.
In the present study a defined medium was developed to determine the carbon and energy source utilisation patterns of 17 clinical isolates of $B$. bronchiseptica from various animal sources and representing the three phase types [13-14]. The effect of carbon source on cell surface properties was also examined.

\section{Materials and methods}

\section{Bacterial strains}

The 17 clinical isolates of $B$. bronchiseptica used for this study were obtained, grown and stored as described previously [13]. These isolates included: four smooth phase (SP) organisms from dogs (2) and rats (2); 10 intermediate phase (IP) isolates from dogs (4), guineapig (1), cat (1) and swine (4); and three rough phase (RP) organisms from dog, cat and guinea-pig. Each isolate was stored at $-70^{\circ} \mathrm{C}$ until used. After thawing, the isolates were passaged no more than four times.

\section{Growth conditions and growth rates}

A defined growth medium (DGM) was developed (Table 1) and used for liquid cultures or solidified by the addition of Bacto Agar (Difco) $1.5 \% \mathrm{w} / \mathrm{v}$. The carbohydrates and sugar alcohols (dextrose, mannose, galactose, maltose, mannitol, rhamnose, sorbitol, inositol, fructose, salicin, xylose, lactose, raffinose and sucrose) and organic and amino acids (citrate, succinate $\alpha$-ketoglutarate, malate, fumarate, oxalo-acetate, lactate, acetate, pyruvate, lysine, glutamic acid sodium salt, phenylalanine, arginine, asparagine, tyrosine, histidine, glutamine, tryptophan, aspartic acid sodium 
Table 1. Defined medium for the growth of B. bronchiseptica and testing of carbon source utilisation

\begin{tabular}{lr}
\hline Salts stock solution* & \\
$\mathrm{MgSO}_{4} \cdot 7 \mathrm{H}_{2} \mathrm{O}$ & $10.0 \mathrm{~g}$ \\
$\mathrm{FeSO} \cdot 7 \mathrm{H}_{2} \mathrm{O}$ & $0.5 \mathrm{~g}$ \\
$\mathrm{NaCl}$ & $0.5 \mathrm{~g}$ \\
$\mathrm{MnSO}_{4} \cdot 7 \mathrm{H}_{2} \mathrm{O}$ & $2.0 \mathrm{~g}$ \\
$\mathrm{H}_{2} \mathrm{O}^{\dagger}$ & $250 \mathrm{ml}$ \\
$\mathrm{Phosphate} \mathrm{stock} \mathrm{solution}$ & \\
$\mathrm{KH}_{2} \mathrm{PO}_{4}$ & $2.0 \mathrm{~g}$ \\
$\mathrm{~K}_{2} \mathrm{HPO}_{4}$ & $1.0 \mathrm{~g}$ \\
$\mathrm{H}_{2} \mathrm{O}$ & $100 \mathrm{ml}$ \\
Yeast nitrogen base without amino acids and & \\
ammonium sulphate (YNB) stock solution & \\
$\mathrm{YNB}^{\mathrm{N}}$ & \\
$\mathrm{H}_{2} \mathrm{O}$ & $6.7 \mathrm{~g}$ \\
$\mathrm{Ammonium}$ chloride stock solution & $100 \mathrm{ml}$ \\
$\mathrm{NH}$ & \\
$\mathrm{H}_{2} \mathrm{O}$ & \\
Complete medium (100 ml; pH 7.0$)$ & $15 \mathrm{~g}$ \\
Salts stock solution & $100 \mathrm{ml}$ \\
Phosphate stock solution & \\
Yeast nitrogen base stock solution & $2 \mathrm{ml}$ \\
$\mathrm{Ammonium}$ chloride stock solution & $10 \mathrm{ml}$ \\
$\mathrm{H}_{2} \mathrm{O}$ & $1 \mathrm{ml}$ \\
\hline
\end{tabular}

*Phosphate and ammonium chloride stock solutions were autoclaved, the remaining stock solutions were filter sterilised.

${ }^{\dagger}$ Distilled de-ionised water

salt, cysteine, proline, methionine, glycine, alanine, valine, leucine, isoleucine, serine and threonine) were tested for their ability to act as the sole carbon and energy source for growth. All of the inorganic chemicals were obtained from Fisher Scientific (Pittsburgh, PA, USA). The amino acids and other organic chemicals were obtained from Sigma. All the amino acids used were of the L-configuration and the carbohydrates were of the D-configuration. The yeast nitrogen base without amino acids and ammonium chloride was from Difco. Each of the carbon and energy sources was filter-sterilised, then added aseptically to DGM at $0.02,0.125,0.25,0.5$ and $1.0 \mathrm{~g} / \mathrm{L}$ of DGM (pH 7.0). Growth was determined by an increase in absorbance (A) and colony count after incubation $\left(37^{\circ} \mathrm{C}\right.$; shaking culture; initial $\mathrm{A}_{540}$ of 0.01 ) for at least $72 \mathrm{~h}$. Ability to utilise a carbon source was defined as growth after three consecutive transfers $(0.005-0.02 \mathrm{ml}$ of inoculum $/ 5 \mathrm{ml}$ of DGM; $\mathrm{A}_{540}$ equivalent of 0.01 ) through DGM containing the carbon source. Brucella Broth or Agar (Difco) was used as the growth control.

Growth rates for representatives of each phase type isolate $110 \mathrm{H}$ (SP), 87 (IP) and 110NH (RP) - were determined by standard techniques, i.e., from the change in $\mathrm{A}_{540}$ and viable cell count in cultures in their second transfer through DGM. All experiments were done in triplicate and repeated with different batches of defined media, and results expressed as means and SEM.

\section{Phase switching (dissociation)}

The effect of carbon and energy source on the stability of the phase type of the $B$. bronchiseptica isolates was determined. The criteria used to assess phase dissociation were colonial morphology and motility [13]. Isolates were inoculated on to DGM agar with a carbon and energy source $(1.0 \mathrm{mg} / \mathrm{ml})$, or Brucella agar as the control. Colonial morphology was determined by examination of 100 colonies on each agar plate (five plates total) at random, with a dissecting microscope and indirect illumination [13]. Motility was assessed by microscopic observation of a hanging drop wet mount preparation.

\section{Cell-surface properties}

Isolates in the late logarithmic stage of growth on the various carbon sources were examined by transmission electron microscopy for the presence of pili and flagella. Bacteria were grown overnight in either Brucella broth or in DGM with a carbon and energy source $(1.0 \mathrm{mg} / \mathrm{ml}$ of DGM). Samples $(0.1 \mathrm{ml})$ were placed on a collodion-coated copper grid and stained with phosphotungstic acid $0.2 \%, \mathrm{pH} 7.2$. Because of the peritrichous arrangement of the flagella and pili, the degree of piliation and flagellation per cell was estimated for 50 bacterial cells by counting only the pili and flagella that extended from the edge of the cell in the plane of the collodion support. In addition, isolates $110 \mathrm{H}, 87$ and $110 \mathrm{NH}$, representative of the three phase types (SP, IP and RP, respectively), grown on the various carbon sources, were assessed for their piliation and flagellation at different stages in the growth cycle (early logarithmic, late logarithmic and mid-stationary - mid-point between initiation of stationary phase plateau and start of decline in viability).

The ability of strains $110 \mathrm{H}, 87$ and $110 \mathrm{NH}$ to produce capsule in response to specific sources of carbon and energy was determined by the standard method of light microscopic examination of negatively stained (nigrosin) cell suspensions. They were grown overnight on DGM agar containing a carbon source $(1.0 \mathrm{mg} / \mathrm{ml})$. and on Brucella agar. Determination of the degree of capsulation was made on encoded preparations to avoid bias. A non-capsulate strain of Escherichia coli was used as the negative control and a capsulate strain of Klebsiella pneumoniae was the positive control for determination of capsule formation.

The effect of carbon and energy source on adherence of $B$. bronchiseptica isolates to hamster lung fibroblasts was determined as described previously [15]. Organisms in early logarithmic, late logarithmic and mid-stationary phases of growth were pelleted, then

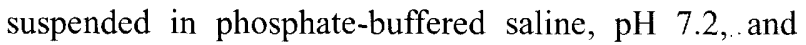
incubated for $30 \mathrm{~min}$ at $37^{\circ} \mathrm{C}$ with hamster lung fibroblasts (Don Line) in monolayer culture on coverslips. After incubation, the monolayers were washed free of non-adherent bacteria, fixed and stained with crystal violet $1 \% \mathrm{w} / \mathrm{v}$. The number of adherent 
bacteria/fibroblast for 50 fibroblasts/coverslip was counted. Each experiment was done three times in triplicate.

\section{Results}

Carbon sources sustaining growth and their effect on colonial morphology

Carbon and energy requirements for growth of the 17 isolates of B. bronchiseptica, regardless of host species, were met by four of the 20 amino acids (glutamate, glutamine, proline and tyrosine) and all the other organic acids tested (succinate, citrate, $\alpha$-ketoglutarate, acetate, fumarate, pyruvate, malate, lactate and oxaloacetate). None of the carbohydrates or sugar alcohols tested (dextrose, mannose, galactose, maltose, mannitol, rhamnose, sorbitol, inositol, fructose, salicin, xylose, lactose, raffinose and sucrose) supported growth.

Changes in colonial morphology of IP and RP isolates consistent with phase dissociation were not observed on DGM containing any of the organic acids, even through repeated laboratory passage. The level of phase dissociation for the SP isolate $110 \mathrm{H}$ to the rough phase was not significantly different from that reported for this isolate after growth on Brucella agar [13]. Thus, spontaneous dissociation of SP colonies to RP colonies was not inhibited or enhanced by the cultural conditions tested. No back-revertants (roughto-smooth colonies) were detected, a finding which confirms a previous observation that the IP and RP isolates are stable colonial morphologies [13].

Comparison of generation times for smooth $(110 \mathrm{H})$, intermediate (87) and rough (110NH) phase isolates, during growth in DGM containing various carbon sources, showed that doubling times varied with both the isolate and the carbon source tested (Table 2). Overall, the fastest doubling times were for isolates grown in Brucella broth $(0.82 \mathrm{~h}$, isolate $110 \mathrm{H} ; 1.4 \mathrm{~h}$, isolate $87 ; 0.99 \mathrm{~h}$, isolate $110 \mathrm{NH}$ ). With respect to the single carbon and energy sources, there was a considerable range in generation times measured $(1.9-6.9 \mathrm{~h}$. isolate $110 \mathrm{H} ; 1.7-5.2 \mathrm{~h}$, isolate $87 ; 1.4-$ $4.5 \mathrm{~h}$, isolate $110 \mathrm{NH})$. However, final cell concentrations for all isolates grown in DGM were similar to those measured for the same isolates grown in Brucella broth $\left(10^{8-9}\right.$ bacteria $/ \mathrm{ml}$ of medium).

\section{Influence of growth conditions on cell surface properties}

The effect of carbon and energy source on the production of capsule was assessed. The relative amounts of capsulation varied with the source of carbon and energy for isolates 87 and $110 \mathrm{H}$, but not for isolate $110 \mathrm{NH}$, as shown in Table 2 .

Bacteria from the late logarithmic growth stage were assessed for the presence of pili and flagella by electron microscopy and negatively stained preparations. Regardless of carbon source, all 17 isolates displayed the pattern of piliation and flagellation typical for their respective phase type, with patterns and numbers of appendages similar to those observed for controls grown in Brucella broth. The extent of piliation and flagellation of the isolates representing the three colonial phase types when grown in Brucella broth is shown in Table 3. When present, both flagella and pili were distributed peritrichously on the bacterial cell surface. Piliation of strains 87 (IP) and $110 \mathrm{NH}$ (RP) was dependent on the age of the culture. The extent of piliation of the smooth phase isolates throughout their growth cycle, as exemplified by isolate $110 \mathrm{H}$, exceeded that expressed by either rough or intermediate phase isolates.

Adherence to hamster lung fibroblasts of two of the three phase types varied with culture age, as exemplified by the results presented for isolates 87

Table 2. Effect of single sources of carbon and energy on mean generation time (MGT) and capsule production of $B$. bronchiseptica isolates

\begin{tabular}{|c|c|c|c|c|c|c|}
\hline \multirow[b]{2}{*}{ Carbon source ${ }^{*}$} & \multicolumn{2}{|c|}{ Smooth phase (isolate $110 \mathrm{H}$ ) } & \multicolumn{2}{|c|}{ Intermediate phase (isolate 87 ) } & \multicolumn{2}{|c|}{ Rough phase (isolate $110 \mathrm{NH}$ ) } \\
\hline & MGT (h) (SEM) & Capsule $^{\dagger}$ & MGT (h) (SEM) & Capsule $^{\dagger}$ & MGT (h) (SEM) & Capsule $^{\dagger}$ \\
\hline Brucella broth (control) & $0.82(0.01)$ & ++ & $1.4(0.02)$ & ++ & $0.99(0.02)$ & + \\
\hline Succinate & $2.1 \quad(0.01)$ & ++ & $1.7(0.01)$ & ++ & $1.4 \quad(0.02)$ & + \\
\hline Citrate & $1.9 \quad(0.01)$ & + & $1.8(0.01)$ & ++++ & $1.6 \quad(0.01)$ & + \\
\hline$\alpha$-Ketoglutarate & $2.2 \quad(0.01)$ & ++ & $1.8(0.01)$ & ++ & $1.5 \quad(0.02)$ & + \\
\hline Acetate & $2.4 \quad(0.02)$ & + & $2.0(0.01)$ & + & $2.3 \quad(0.01)$ & + \\
\hline Fumarate & $2.7 \quad(0.02)$ & + & $2.1(0.02)$ & + & $2.0 \quad(0.02)$ & + \\
\hline Pyruvate & $3.0 \quad(0.02)$ & +++ & $3.0(0.01)$ & +++ & $3.0 \quad(0.03)$ & + \\
\hline Malate & $2.8 \quad(0.01)$ & + & $3.6(0.01)$ & ++ & $2.8 \quad(0.01)$ & + \\
\hline Lactate & $5.7 \quad(0.02)$ & + & $3.6(0.03)$ & + & $4.2 \quad(0.01)$ & + \\
\hline Oxalo-acetate & $3.8 \quad(0.01)$ & + & $4.0(0.02)$ & + & $4.5 \quad(0.01)$ & + \\
\hline Tyrosine & $2.6 \quad(0.01)$ & + & $1.8(0.01)$ & + & $2.1 \quad(0.03)$ & + \\
\hline Proline & $4.6 \quad(0.02)$ & ++ & $2.7(0.01)$ & ++ & .. $3.3 \quad(0.02)$ & + \\
\hline Glutamate & $3.6 \quad(0.01)$ & ++ & $3.5(0.01)$ & +++ & $3.7 \quad(0.01)$ & + \\
\hline Glutamine & $6.9 \quad(0.03)$ & ++++ & $5.2(0.03)$ & + & $4.1 \quad(0.03)$ & + \\
\hline
\end{tabular}

${ }^{*}$ All single carbon sources were tested at $1.0 \mathrm{mg} / \mathrm{ml}$.

${ }^{\dagger}$ Relative amounts of capsule observed microscopically. 
Table 3. Effect of stage of growth in Brucella broth on cell surface properties of B. bronchiseptica

\begin{tabular}{|c|c|c|c|c|c|c|c|}
\hline Phase morphotype & $\begin{array}{l}\text { Growth } \\
\text { phase }^{*}\end{array}$ & $\begin{array}{c}\text { Number } \\
\text { of pili/cell }{ }^{\dagger} \\
\text { (range) }\end{array}$ & $\begin{array}{c}\text { Percentage } \\
\text { of bacteria } \\
\text { piliated }\end{array}$ & $\begin{array}{l}\text { Number of } \\
\text { flagella/cell } \\
\text { (range) }\end{array}$ & $\begin{array}{l}\text { Percentage } \\
\text { of bacteria } \\
\text { flagellated }\end{array}$ & Motility & Adherence ${ }^{*}$ \\
\hline \multirow[t]{3}{*}{ Smooth (isolate $110 \mathrm{H}$ ) } & Early logarithmic & $11(1-40)$ & 96 & $0(-)$ & 0 & $(-)$ & $28(2)$ \\
\hline & Late logarithmic & $13(3-45)$ & 98 & $3(1-3)$ & 3 & $(+)$ & $29(2)$ \\
\hline & Mid-stationary & $9(1-20)$ & 82 & $4(3-7)$ & 4 & $(+)$ & $28(1)$ \\
\hline \multirow[t]{3}{*}{ Intermediate (isolate 87 ) } & Early logarithmic & $3(1-3)$ & 24 & $3(1-6)$ & 100 & $(+)$ & $25(3)$ \\
\hline & Late logarithmic & $5(1-6)$ & 54 & $5(1-9)$ & 100 & $(+)$ & $29(1)$ \\
\hline & Mid-stationary & $1(-)$ & 2 & $3(1-6)$ & 34 & $(+)$ & $11(3)$ \\
\hline \multirow[t]{3}{*}{ Rough (isolate $110 \mathrm{NH}$ ) } & Early logarithmic & $0(-)$ & 0 & $3(1-6)$ & 100 & $(+)$ & $3(2)$ \\
\hline & Late logarithmic & $2(1-4)$ & 34 & $3(1-6)$ & 100 & $(+)$ & $19(2)$ \\
\hline & Mid-stationary & $4(1-6)$ & 34 & $3(1-6)$ & 100 & $(+)$ & $29(3)$ \\
\hline
\end{tabular}

* Determined from $\mathrm{A}_{540}$ readings.

Mean results from two experiments. A total of 50 bacteria were surveyed for each time point. Calculation of mean numbers of pili and flagella includes only those cells possessing pili or flagella.

Mean number of adherent bacteria per hamster lung fibroblast (SEM).

Presence or absence of microscopically detected translocational movement in a hanging drop wet mount preparation.

(IP) and $110 \mathrm{NH}$ (RP) (Table 3). The level of adherence of the smooth phase isolates, as shown by strain $110 \mathrm{H}$, was similar at all phases of growth. All organisms of a particular phase type showed similar variations, or not, according to growth stage regardless of carbon source or animal host of origin. Adherence appeared to be related to the level of pilus expression. The finding that early logarithmic cultures of isolate $110 \mathrm{NH}$ exhibited low levels of adherence, despite the lack of observable pili, may be indicative of the level of sensitivity of direct observation, the presence of an alternative adherence mechanism [15], or both.

Rough and intermediate phase isolates were flagellate throughout their growth cycle as shown by isolates $110 \mathrm{NH}$ and 87 , respectively (Table 3 ). In contrast, flagella were not observed on the smooth phase isolate, $110 \mathrm{H}$, until the late logarithmic phase of growth and then only appeared on c. $3 \%$ of the organisms. This observation is consistent with the rate of smooth-to-rough phase switching that has been reported previously [13]. For all isolates, the presence of flagella correlated positively with motility.

\section{Discussion}

Previous studies have reported that in a complex broth medium containing glucose, B. bronchiseptica (phase type unreported) grew aerobically, increasing the $\mathrm{pH}$ of the medium, but that no growth was observed anaerobically in the presence of glucose, lactose, maltose, saccharose, dextrose or mannitol [7]. The use of a defined medium in this study supports and extends previous hypotheses that B. bronchiseptica is asaccharolytic.

The reported ability of $B$. bronchiseptica to utilise carbon sources other than saccharides for growth is not as clear cut. Studies on the nutritional requirements of $B$. bronchiseptica indicated a high level of variability between isolates in their ability to utilise particular carbon sources [9-12]. Proom [9] reported that, whereas most isolates tested could grow in a mixture of amino acids or citrate or lactate, only two 'old laboratory strains' were capable of growing with glutamate, $\alpha$-ketoglutarate, succinate or pyruvate as carbon sources. The findings in the present study, with low passage number isolates of $B$. bronchiseptica, were analogous to those with the laboratory passaged strains of Proom with regard to their ability to utilise some of the organic acids other than amino acids. The range of carbon sources supporting growth was also expanded from Proom's initial work. Furthermore, no variability was observed between isolates from different hosts or of different phase types in their ability to use a particular carbon and energy source for growth. However, growth rates did vary according to isolate and carbon source, although final cell concentration was similar to that after growth in Brucella broth. These findings confirm those of Bemis et al. [13] on 50 strains of $B$. bronchiseptica in that there is remarkably little variability between isolates in some of their physiological characteristics, including colony dissociation rate of smooth phase isolates, haemagglutination pattern, antibiotic susceptibility and nitrate reduction.

None of the isolates exhibited an absolute requirement for glutamic acid, as had been previously reported [9]. Furthermore, glutamine, proline or tyrosine, in addition to glutamic acid, supported growth. B. bronchiseptica had been reported to deaminate serine, glycine, alanine and leucine [10]. However, none of these amino acids was able to support the growth of any of the 17 isolates tested. Thus, there does not appear to be a direct correlation between deaminase activity and substrate utilisation for growth, as was suggested by Rowatt [10].

An effect of carbon source on colonial phenotypes and phase dissociation was not discernible. However, carbon source and growth stage did influence the expression of cell surface structures including capsule, flagella and pili. While these results are in agreement with the findings of Lee et al. [16], that pili are 
ubiquitous in this species, Bemis et al. [13] reported that the intermediate and rough phase organisms were variable with respect to pilus production. The discrepancies that exist in the literature as to the ultrastructural properties of $B$. bronchiseptica may be explained by the different conditions under which $B$. bronchiseptica has been grown [13,15-19]. Thus, the caution expressed by Peppler and Schrumpf [19] on the influence of growth media on antigenic variation in $B$. bronchiseptica is well founded. These cultureinfluenced alterations in cell surface structures could potentially affect host-parasite interactions.

Pili are considered to mediate $B$. bronchiseptica adherence to mammalian cells $[15,17-18]$. Expression of these surface structures was to some extent affected by stage of growth. However, the carbon source did not appear to alter expression of pili as compared to control organisms grown in Brucella broth. Pili were not detected on rough phase organisms in early logarithmic phase. The lack of pili detectable by electron microscopy raises the possibility that, at least for the rough phase isolates, adherence may be mediated via surface structures other than pili. However, it must be cautioned that the level of sensitivity of detection of pili by electron microscopy cannot preclude their possible involvement in adherence to mammalian cells. It has been reported by us and others $[18,20]$ that multiple haemagglutinins are likely to exist within this species, and that there are at least two mechanisms for adherence to hamster lung fibroblasts [15]. Further studies will be needed to determine if the antigenic characteristics of the pili and receptors to which they bind are influenced by growth substrate.

The role of capsule or flagella as virulence factors for B. bronchiseptica has not been defined. Of the surface structures examined, the expression of capsular material was most affected by the carbon source available. However, there appeared to be no correlation between the presence of observable capsule, or amount of capsulation and generation time, stage of growth, or adherence to hamster lung fibroblasts. Bemis et al. [13] reported that capsule was not readily demonstrable on the organisms examined, whereas Nakase $[21,22]$ reported phase I organisms to be both capsulate and flagellate. This conflict may best be attributed, as in the case of piliation, to differences in culture conditions. The findings of the present study confirm those of Bemis et al. [13] that smooth phase isolates can be non-flagellate, although this property is growth-stage dependent, and flagellation is probably a reflection of the number of smooth phase isolates that have undergone dissociation to rough phase isolates in culture.

A culture medium has been developed to assess the influence that carbon substrates have on the growth and surface properties of $B$. bronchiseptica. The study of the biological characteristics of B. bronchiseptica and its virulence factors will lead to an increased understanding of the factors which allow for the broad host range of this organism and variation in its pathogenicity.

\section{References}

1. Goodnow RA. Biology of Bordetella bronchiseptica. Microbiol Rev 1980; 44: 722-738.

2. Woolfrey BF, Moody JA. Human infections associated with Bordetella bronchiseptica. Clin Microbiol Rev 1991; 4: $243-255$.

3. Bauwens JE, Spach DH, Schacker TW, Mustafa MM, Bowden RA. Bordetella bronchiseptica pneumonia and bacteremia following bone marrow transplantation. $J$ Clin Microbiol 1992; 30: 2474-2475.

4. Woodard DR, Cone LA, Fostvedt K. Bordetella bronchiseptica infection in patients with AIDS. Clin Infect Dis 1995; 20: 193-194

5. Porter JF, Parton R, Wardlaw AC. Growth and survival of Bordetella bronchiseptica in natural waters and in buffered saline without added nutrients. Appl Environ Microbiol 1991; 57: $1202-1206$.

6. Porter JF, Wardlaw AC. Long-term survival of Bordetella bronchiseptica in lakewater and in buffered saline without added nutrients. FEMS Microbiol Lett 1993; 110: 33-36.

7. Ferry NS. Etiology of canine distemper. J Infect Dis 1911; 8: 399-420.

8. Ferry NS, Noble A. Studies relative to the apparent close relationship between Bact. pertussis and B. bronchisepticus. I. Cultural agglutination and absorption reactions. $J$ Bacteriol 1918; 3: 193-208.

9. Proom $H$. The minimal nutritional requirements of organisms of the genus Bordetella Lopez. J Gen Microbiol 1955; 12: $63-75$.

10. Rowatt E. Amino acid metabolism in the genus Bordetella. $J$ Gen Microbiol 1955; 13: 552-560.

11. Snell JJS, Lapage SP. Carbon source utilization tests as an aid to the classification of non-fermenting gram-negative bacteria. $J$ Gen Microbiol 1973; 74: 9-20.

12. Ulrich JA, Needham GM. Differentiation of Alcaligenes faecalis from Brucella bronchisepticus by biochemical and nutritional methods. $J$ Bacteriol 1953; 65: 210-215.

13. Bemis DA, Greisen HA, Appel MJG. Bacteriological variation among Bordetella bronchiseptica isolates from dogs and other species. J Clin Microbiol 1977; 5: 471-480.

14. Bemis DA, Greisen HA, Appel MJG. Pathogenesis of canine bordetellosis. J Infect Dis 1977; 135: 753-762.

15. Plotkin BJ, Bemis DA. Adherence of Bordetella bronchiseptica to hamster lung fibroblasts. Infect Immun 1984; 46: 697-702.

16. Lee SW, Way AW, Osen EG. Purification and subunit heterogeneity of pili of Bordetella bronchiseptica. Infect Immun 1986; 51: 586-593.

17. Bemis DA, Wilson SA. Influence of potential virulence determinants on Bordetella bronchiseptica-induced ciliostasis. Infect Immun 1985; 50: 35-42.

18. Ishikawa $\mathrm{H}$, Isayama $\mathrm{Y}$. Bovine erythrocyte-agglutinin as a possible adhesin of Bordetella bronchiseptica responsible for binding to porcine nasal epithelium. J Med Microbiol 1988; 26: 205-209.

19. Peppler MS, Schrumpf ME. Phenotypic variation and modulation in Bordetella bronchiseptica. Infect Immun 1984; 44: $681-687$

20. Bemis DA, Plotkin, BJ. Hemagglutination by Bordetella bronchiseptica. J Clin Microbiol 1982; 15: 1120-1127.

21. Nakase Y. Studies on Hemophilus bronchisepticus I. The antigenic structures of Hemophilus bronchisepticus from guinea pig. Kitasato Arch Exp Med 1957; 30: 57-72.

22. Nakase Y. Studies on Hemophilus bronchisepticus II. Phase variation of Hemophilus bronchisepticus. Kitasato Arch Exp Med 1957; 30: 73-78. 